\title{
$\mathbf{N}$ 掺杂 $\mathbf{T i O}_{2}$ 纳米粒子表面光生电荷特性与光催化活性
}

\author{
张晓茹＼cjkstart林艳红 * 张健夫何冬青王德军 \\ (吉林大学化学学院, 长春 130012 )
}

\begin{abstract}
摘要：以尿素为氮源, 采用水热法制备了不同 $\mathrm{N}$ 掺杂量的 $\mathrm{TiO}_{2}\left(\mathrm{~N}-\mathrm{TiO}_{2}\right)$ 光催化剂. 利用 $\mathrm{X}$ 射线衍射 $(\mathrm{XRD})$, 紫 外-可见漫反射光谱 (UV-Vis DRS), X 射线光电子能谱(XPS)及苂光(PL)光谱等技术对其进行了系统的表征. 以 罗丹明 $\mathrm{B}(\mathrm{RhB})$ 和甲基橙(MO)溶液的脱色降解为模型反应, 分别考察了 $\mathrm{N}^{-} \mathrm{TiO}_{2}$ 光催化剂在紫外和可见光区的 光催化活性. 利用表面光伏(SPV)和瞬态光伏(TPV)技术研究了 $\mathrm{N}-\mathrm{TiO}_{2}$ 纳米粒子表面光生电荷的产生和传输机 制, 并探讨了光生电荷与光催化活性之间的关系. 结果显示, 随着 $\mathrm{N}$ 含量的增大, $\mathrm{TiO}_{2}$ 表面光伏响应阈值红移, 可见光部分光电压响应强度逐渐增强, 瞬态光伏响应达到最大值的时间亦有着不同程度的延迟. 这表明适量的 $\mathrm{N}$ 掺杂能够提高 $\mathrm{TiO}_{2}$ 纳米粒子中光生载流子的分离效率, 相应地延长载流子的传输时间, 增加光生电荷的寿命, 从而促进其光催化活性; 而过量的 $\mathrm{N}$ 掺杂则增加了 $\mathrm{TiO}_{2}$ 纳米粒子中光生载流子的复合中心, 抑制其光催化活性.
\end{abstract}

关键词： $\mathrm{N}$ 掺杂 $\mathrm{TiO}_{2}$ ；表面光伏；瞬态光伏；光生电荷；光催化活性

中图分类号： O643; O644

\section{Photoinduced Charge Carrier Properties and Photocatalytic Activity of N-Doped $\mathrm{TiO}_{2}$ Nanocatalysts}

\author{
ZHANG Xiao-Ru LIN Yan-Hong* ZHANG Jian-Fu HE Dong-Qing $\quad$ WANG De-Jun \\ (College of Chemistry, Jilin University, Changchun 130012, P. R. China)
}

\begin{abstract}
Nitrogen-doped $\mathrm{TiO}_{2}\left(\mathrm{~N}-\mathrm{TiO}_{2}\right)$ photocatalysts with different amounts of $\mathrm{N}$ doping were successfully synthesized by the hydrothermal method using urea as the nitrogen source. The samples were characterized by X-ray diffraction (XRD), UV-Vis diffuse reflectance spectroscopy (UV-Vis DRS), X-ray photoelectron spectroscopy (XPS), and photoluminescence (PL) spectroscopy. The photodegradation of rhodamine B (RhB) and methyl orange (MO) solutions was used to evaluate the photocatalytic activity of the catalysts under UV and visible light irradiation. Surface photovoltage (SPV) and transient photovoltage (TPV) techniques were used to investigate the separation and transport mechanism of the photogenerated charge carriers of the $\mathrm{N}$-doped $\mathrm{TiO}_{2}$ nanoparticles. The relationship between the photogenerated charge carriers and photocatalytic activity was also discussed. The results showed that the SPV threshold values shifted to the visible region and a stronger photovoltaic response in the visible region was observed with an increase in $\mathrm{N}$ doping. We also found that the maximum TPV response time was different for $\mathrm{N}_{-} \mathrm{TiO}_{2}$. These results indicate that with an appropriate amount of $\mathrm{N}$ doping, the photoinduced charge carriers separate efficiently, the transmission time increases, and the lifetime of the photoinduced charge carriers increases. Therefore, the photocatalytic activity is enhanced. However, excessive $\mathrm{N}$ acts as recombination centers for photoinduced electrons and holes, which reduces their photocatalytic activity.
\end{abstract}

Key Words: $\quad \mathrm{N}$-doped $\mathrm{TiO}_{2} ; \quad$ Surface photovoltage; Transient photovoltage; Photo-induced charge carrier; Photocatalytic activity

Received: May 21, 2010; Revised: July 16, 2010; Published on Web: August 24, 2010.

*Corresponding author. Email: linyh@jlu.edu.cn; Tel: +86-431-85168093.

The project was supported by the National Key Basic Research Program of China (973) (2007CB613303), National Natural Science Foundation of China (20703020, 20873053), and Scientific Forefront and Interdisciplinary Innovation Project, Jilin University, China (421031401412). 国家重点基础研究发展规划项目(973) (2007CB613303), 国家自然科学基金(20703020, 20873053)和吉林大学科学前沿与交叉学科创新项目 (421031401412)资助 
近年来以纳米 $\mathrm{TiO}_{2}$ 为代表的传统半导体材料, 由于其价廉、无毒、化学性质稳定等特点而成为具有 广阔应用前景的光催化剂 ${ }^{[1-4]}$. 然而, 由于 $\mathrm{TiO}_{2}$ 的带 隙较宽(锐钛矿, $3.2 \mathrm{eV}$ ), 只能被紫外光激发, 而且其 光生电子和空穴容易复合, 导致太阳光的利用率和 量子转换效率较低, 极大地限制了其在环境净化方 面的实际应用. 如何拓展 $\mathrm{TiO}_{2}$ 的光响应范围, 提高 光催化效率也因此成为光催化领域最具挑战性的课 题之一. 自从 2001 年 Asahi 等人 ${ }^{[5]}$ 提出了 $\mathrm{N}$ 掺杂 $\mathrm{TiO}_{2}$ 能够使其光吸收范围扩展到可见光区域以来, 基于 $\mathrm{N}$ 掺杂 $\mathrm{TiO}_{2}$ 光催化剂的研究和开发引起了人 们的广泛关注 ${ }^{[-9]}$. 影响光催化过程中太阳能利用率 的关键在于所选半导体材料的能级结构, 外来离子 的掺人能够改变 $\mathrm{TiO}_{2}$ 的能级结构, 从而引起其光电 性质的重大改变 ${ }^{[10-12]}$. 通常, 半导体材料在光的激发 下 $\left(h \nu>E_{\mathrm{g}}, E_{\mathrm{g}}\right.$ 为带隙宽度), 光生载流子(电子、空穴) 迁移到表面参与氧化还原反应, 在此过程中, 可能影 响光催化过程量子效率的重要因素有光生载流子的 分离效率和复合效率, 以及光生载流子向表面迁移 过程中载流子的迁移率等 ${ }^{[13-14]}$. 因此深人研究 $\mathrm{N}$ 掺 杂 $\mathrm{TiO}_{2}$ 纳米结构中光生电荷的激发、分离、迁移及 复合等复杂的物理化学过程, 对于提高光催化效率, 揭示表面光催化反应本质具有重大的理论和实际意 义.

表面光伏(SPV)和瞬态光伏(TPV)技术是研究纳 米半导体材料表面光生电荷行为的有效手段, SPV 可以得到纳米材料体系中光生电荷的基本性质; 而 $\mathrm{TPV}$ 可以分析纳米结构材料的光生电荷的动力学 特性, 在国际上利用其对光电体系的研究也取得了 较好进展 ${ }^{[15-17]}$, 但对 $\mathrm{N}$ 掺杂 $\mathrm{TiO}_{2}$ 的表面光生电荷特 性的研究尚未见报道. 本文通过水热法制备了不同 $\mathrm{N}$ 掺杂量的 $\mathrm{TiO}_{2}$ 纳米粒子, 利用 XRD、XPS、UVVis DRS 等技术对样品的晶型结构、掺杂状态以及 可见光吸收特性进行了表征, 并考察了紫外光和可 见光下不同 $\mathrm{N}$ 掺杂量对其光催化活性的影响. 重点 是利用 SPV 和 TPV 技术对这一系列 $\mathrm{N}$ 掺杂 $\mathrm{TiO}_{2}$ 光催化剂光生电荷的分离、传输和复合行为进行研 究, 并讨论光生电荷的微观运动行为对紫外光及可 见光催化性能的影响.

\section{1 实验部分}

\section{1 主要试剂与仪器}

钛酸四丁酯(TBOT, 化学纯, $>98.0 \%$, 国药集团
化学试剂有限公司), 无水乙醇 (优级纯, >99.5\%), 尿 素(分析纯, >99.0\%), 浓硝酸(分析纯), 均为北京化 工厂产品.

$X$ 射线衍射仪(XRD, Max-2550, 日本 Rigaku 公 司), $\mathrm{Cu}$ 靶, 电压 $40 \mathrm{kV}$, 电流 $30 \mathrm{~mA}$, 扫描速率为 5 $\left(^{\circ}\right) \cdot \mathrm{min}^{-1}$; 紫外-可见漫反射光谱仪(UV-Vis DRS, UV2450, 日本 Shimadzu 公司); X 射线光电子能谱仪 (XPS, Escalab 250, 美国 Thermo VG Scientific 公司), 以 $\mathrm{C} 1 s(284.6 \mathrm{eV})$ 为基准进行结合能校正. 表面光 伏和瞬态光伏分别由自组装的光电压谱仪测定 ${ }^{[15-16]}$, 测量电极为三明治结构(ITO/样品/ITO), 其中表面光 伏测试所用的光源为 $500 \mathrm{~W}$ 的氙灯, 瞬态光伏的光 源为 Nd: YAG 激光器(Polaris II, New Wave Research, Inc.), $355 \mathrm{~nm}$, 脉冲半宽为 $5 \mathrm{~ns}$.

\section{$1.2 \mathrm{~N}$ 掺杂 $\mathrm{TiO}_{2}$ 的制备}

样品的合成参见文献 ${ }^{[18]}$. 首先通过溶胶-凝胶法 合成 $\mathrm{TiO}_{2}$ 干凝胶: 将 $4 \mathrm{~mL}$ 钛酸四丁酯在剧烈摚拌 下加人到 $2 \mathrm{~mL}$ 无水乙醇中, 搅拌 $30 \mathrm{~min}$, 再将 0.4 $\mathrm{mL}$ 浓硝酸、 $1 \mathrm{~mL}$ 二次蒸馏水和 $17 \mathrm{~mL}$ 无水乙醇的 混合溶液滴加到上述溶液中, 继续搅拌直至得到透 明溶胶, 然后将此溶胶在 $110{ }^{\circ} \mathrm{C}$ 干燥 $6 \mathrm{~h}$, 得 $\mathrm{TiO}_{2}$ 干 凝胶. 以尿素为氮源, 将尿素与 $\mathrm{TiO}_{2}$ 干凝胶按不同 的质量比(2:1, 3:1, 4:1)混合研磨, 然后将其加人到适 量的水中进行超声分散, 将所得混合液放人内衬聚 四氟乙烯的高压釜中, 在 $240{ }^{\circ} \mathrm{C}$ 反应 $10 \mathrm{~h}$. 反应产物 经 $110{ }^{\circ} \mathrm{C}$ 干燥后, 在 $200{ }^{\circ} \mathrm{C}$ 焙烧 $6 \mathrm{~h}$, 即可得到不同 $\mathrm{N}$ 掺杂量的 $\mathrm{TiO}_{2}$ 纳米粒子. 按照尿素与 $\mathrm{TiO}_{2}$ 干凝 胶的质量比从小到大的顺序 (2:1, 3:1, 4:1), 样品分别 标记为 $1 \mathrm{~N}-\mathrm{TiO}_{2}, 2 \mathrm{~N}-\mathrm{TiO}_{2}, 3 \mathrm{~N}-\mathrm{TiO}_{2}$.

\section{3 光催化活性测定}

光催化反应在自制的石英瓶 $(50 \mathrm{~mL})$ 中进行. 紫 外光催化剂活性实验通过 $\mathrm{RhB}$ 溶液进行评价, 光源 采用具有冷凝装置的 $500 \mathrm{~W}$ 的高压录灯. 称取 25 $\mathrm{mg} \mathrm{N}-\mathrm{TiO}_{2}$ 粉末置于石英瓶中, 再加人 $20 \mathrm{~mL}$ 初始 浓度为 $20 \mathrm{mg} \cdot \mathrm{L}^{-1}$ 的 $\mathrm{RhB}$ 溶液, 超声 $5 \mathrm{~min}$, 避光磁 力搅拌 $1 \mathrm{~h}$, 使之达到吸附-脱附平衡, 之后置于光源 下进行反应, 每隔 $15 \mathrm{~min}$ 取样 $1 \mathrm{~mL}$, 经离心分离后 取上层清液, 用 723 型可见分光光度计测量 $\mathrm{RhB}$ 溶 液的吸光度, 其最大吸收波长是 $553 \mathrm{~nm}$. 可见光催 化剂活性实验采用 $\mathrm{MO}$ 溶液为降解物, 其初始浓度 同样为 $20 \mathrm{mg} \cdot \mathrm{L}^{-1}$, 光源为 $500 \mathrm{~W}$ 氙灯, 在样品和光 源之间放置一个 $420 \mathrm{~nm}$ 的滤光片, 以滤掉波长小于 $420 \mathrm{~nm}$ 的紫外光. 吸附平衡后, 置于光源下进行反 
应, 每隔 $1 \mathrm{~h}$ 取样 $1 \mathrm{~mL}$, 经离心分离后取上层清液, 用 723 型可见分光光度计测量 $\mathrm{MO}$ 溶液的吸光度, 其最大吸收波长为 $464 \mathrm{~nm}$.

\section{2 结果与讨论}

\section{1 催化剂的表征}

图 1 为纯 $\mathrm{TiO}_{2}$ 和不同 $\mathrm{N}$ 掺杂量的 $\mathrm{TiO}_{2}$ 样品的 XRD 谱图. 各样品的 XRD 图谱与锐钛矿结构 $\mathrm{TiO}_{2}$ 的标准图(JCDPS 卡片 21-1272)完全吻合, 表明 $\mathrm{TiO}_{2}$ 的晶相并没有因为 $\mathrm{N}$ 的引人而发生改变. 同时 XRD 结果显示没有其它杂相存在, 说明样品的纯度 高, 掺人的 $\mathrm{N}$ 没有形成新的物质. 采用 Scherrer 方 程通过衍射峰的宽度计算出样品的平均粒径, 方程 如下: $D=K \lambda /(\beta \cos \theta)$, 式中 $D$ 为晶粒尺寸, $\beta$ 为衍射 峰半高宽, $\theta$ 为该峰对应的衍射角, 人射线波长 $\lambda=$ $0.15405 \mathrm{~nm}$, Scherrer 常数 $K=0.89$. 计算结果显示样 品的粒径均在 $13 \mathrm{~nm}$ 左右, 表面 $\mathrm{N}$ 的掺人对颗粒的 尺寸影响较小.

为了研究掺杂的 $\mathrm{N}$ 原子在 $\mathrm{TiO}_{2}$ 晶格中的化学 态, 本文对样品进行了 XPS 分析. 图 2 为样品表面 $\mathrm{Ti} 2 p, \mathrm{O} l s$ 和 $\mathrm{N} l s$ 的 XPS 图谱. 从 Ti $2 p$ 的 XPS 可 以看出, 各样品测得的 Ti $2 p$ 均由两峰组成, 峰形都 呈比较标准的对称形, 分别对应于 Ti $2 p_{3 / 2}$ 和 Ti $2 p_{1 / 2}$ 的电子结合能, 纯 $\mathrm{TiO}_{2}$ 两峰的电子结合能之差为 $5.8 \mathrm{eV}$, 随着 $\mathrm{N}$ 的掺人, 两峰的电子结合能之差逐渐 变小, 同时, $\mathrm{Ti} 2 p_{3 / 2}$ 和 $\mathrm{O} 1 \mathrm{~s}$ 处的电子结合能也均逐 渐降低. 这是因为 $\mathrm{N}$ 掺人 $\mathrm{TiO}_{2}$ 中后, $\mathrm{N}$ 与 $\mathrm{O}$ 相比电 负性较小, 电子可能从 $\mathrm{N}$ 转移到 $\mathrm{Ti}$, 使 $\mathrm{Ti}$ 周围的电 子云密度发生变化, 导致 Ti $2 p$ 两峰的电子结合能 之差, $\mathrm{Ti} 2 p_{3 / 2}$ 及 $\mathrm{O} l s$ 的电子结合能均变小, 表明 $\mathrm{N}$ 已经进人 $\mathrm{TiO}_{2}$ 晶格中 ${ }^{[19-20]}$. 从 $\mathrm{N} 1 s$ 的XPS 谱图中可 以看到, 在电子结合能 $399.5 \mathrm{eV}$ 处出现了较强的 $\mathrm{N}$

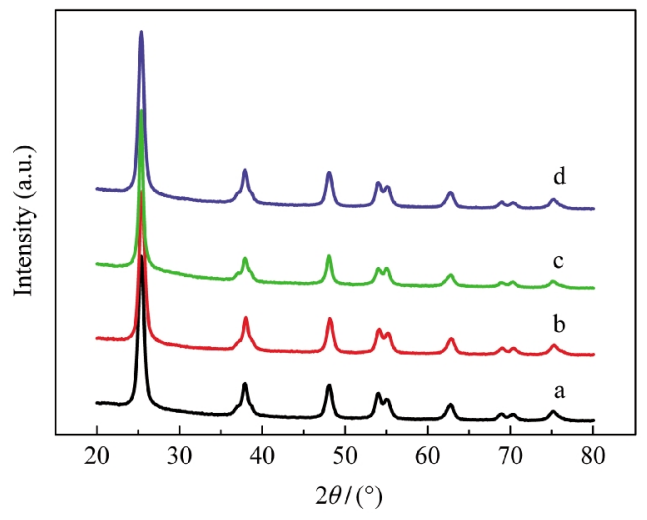

图 1 不同 $\mathrm{N}$ 掺杂量的 $\mathrm{N}-\mathrm{TiO}_{2}$ 纳米粒子的 XRD 谱图

Fig.1 XRD patterns of $\mathrm{N}_{-1} \mathrm{TiO}_{2}$ nanoparticles with different $\mathbf{N}$-doping contents

(a) pure $\mathrm{TiO}_{2}$ nanopowders, (b) $1 \mathrm{~N}-\mathrm{TiO}_{2}$, (c) $2 \mathrm{~N}-\mathrm{TiO}_{2}$, (d) $3 \mathrm{~N}-\mathrm{TiO}_{2}$; The mass ratio of urea $v s \mathrm{TiO}_{2}$ xerogel is 2:1, 3:1, and 4:1 for $1 \mathrm{~N}-\mathrm{TiO}_{2}$, $2 \mathrm{~N}-\mathrm{TiO}_{2}$, and $3 \mathrm{~N}-\mathrm{TiO}_{2}$, respectively.

$1 s$ 特征峰, 表明 $\mathrm{N}$ 原子处于 $\mathrm{O}-\mathrm{Ti}-\mathrm{N}$ 环境中 ${ }^{[5,21]}$. 因 此, 通过 XPS 的测试, 可以确认样品在制备过程中, 尿素中的 $\mathrm{N}$ 原子已经成功在 $\mathrm{TiO}_{2}$ 晶格中取代了部 分 $\mathrm{O}$ 原子. 另外, 根据密度泛函理论(DFT)计算, $\mathrm{N}$ 的掺杂导致形成氧空位所需能量减小, 从而推断 $\mathrm{N}$ 掺杂过程伴随有氧空位的形成 ${ }^{[22]}$. 表 1 为不同 $\mathrm{N}$ 掺 杂量的 $\mathrm{N}-\mathrm{TiO}_{2}$ 光催化剂 XPS 元素分析结果, 可以 得到样品 $1 \mathrm{~N}-\mathrm{TiO}_{2} 、 2 \mathrm{~N}-\mathrm{TiO}_{2} 、 3 \mathrm{~N}-\mathrm{TiO}_{2}$ 中 $\mathrm{N}$ 元素的含 量分别为 $0.50 \% 、 0.71 \% 、 1.10 \%$ ( $x$, atomic fraction).

图 3 给出了样品的 UV-Vis DRS 光谱. 由图可 见, 与纯 $\mathrm{TiO}_{2}$ 相比, 随着 $\mathrm{N}$ 的掺人, 紫外区吸光性 能稍微减弱, 而在可见区的吸光性能明显增强. 其原 因可能是由于 $\mathrm{N}$ 的引人, 在 $\mathrm{TiO}_{2}$ 的 $\mathrm{O} 2 p$ 价带顶之 上形成了局部中间带隙 $(\mathrm{N} 2 p)$ 能级, 该掺杂能级上 的电子可以被激发到 $\mathrm{TiO}_{2}$ 的导带, 使得长波光子也 能被吸收, 因而扩展了 $\mathrm{TiO}_{2}$ 吸收光谱的吸收范围, 提高可见光区的吸收性能.
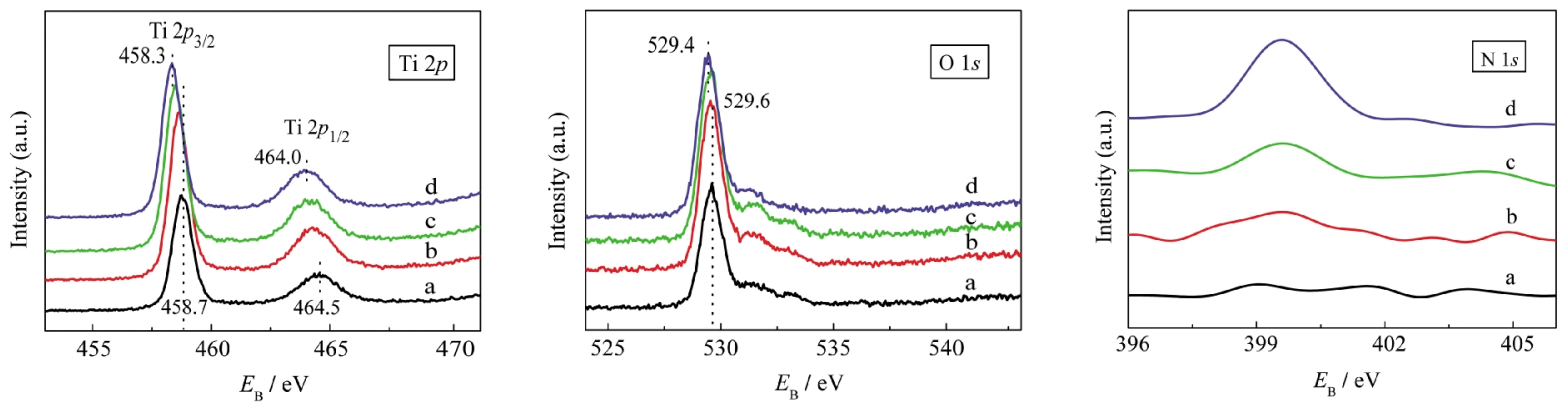

图 2 不同 $\mathbf{N}$ 掺杂量的 $\mathrm{N}^{-\mathrm{TiO}_{2}}$ 纳米粒子的 XPS 谱图

Fig.2 XPS spectra of $\mathrm{N}^{-T i O}{ }_{2}$ nanoparticles with different $\mathrm{N}$-doping contents

(a) $\mathrm{TiO}_{2}$, (b) $1 \mathrm{~N}-\mathrm{TiO}_{2}$, (c) $2 \mathrm{~N}-\mathrm{TiO}_{2}$, (d) $3 \mathrm{~N}-\mathrm{TiO}_{2}$ 
表 1 不同 $\mathbf{N}$ 掺杂量的 $\mathbf{N}^{-T i O} \mathbf{O}_{2}$ 光催化剂 XPS 元素分析结果

Table 1 XPS elemental analysis results of $\mathrm{N}^{-\mathrm{TiO}_{2}}$ photocatalysts with different $\mathrm{N}$-doping contents

\begin{tabular}{cclccc}
\hline Sample & $x_{\mathrm{Ti}}(\%)$ & $x_{\mathrm{N}}(\%)$ & $\begin{array}{c}\mathrm{N} l s \text { peak } \\
\text { position }(\mathrm{eV})\end{array}$ & $\begin{array}{c}\mathrm{Ti} 2 p_{312} \text { peak } \\
\text { position }(\mathrm{eV})\end{array}$ & $\begin{array}{c}\mathrm{O} \text { l } s \text { peak } \\
\text { position }(\mathrm{eV})\end{array}$ \\
\hline $\mathrm{TiO}_{2}$ & 24.50 & 0 & - & 458.7 & 529.6 \\
$1 \mathrm{~N}-\mathrm{TiO}_{2}$ & 24.09 & 0.50 & 399.5 & 458.6 & 529.5 \\
$2 \mathrm{~N}-\mathrm{TiO}_{2}$ & 23.30 & 0.71 & 399.5 & 458.4 & 529.5 \\
$3 \mathrm{~N}-\mathrm{TiO}_{2}$ & 27.45 & 1.10 & 399.5 & 458.3 & 529.4 \\
\hline
\end{tabular}

\section{2 催化剂的光催化活性}

图 $4 \mathrm{a}$ 为 $\mathrm{N}-\mathrm{TiO}_{2}$ 光催化剂的紫外光催化活性测 试结果. 从图中可以看出, $\mathrm{RhB}$ 溶液经过 $50 \mathrm{~min}$ 紫 外光催化降解后, $3 \mathrm{~N}-\mathrm{TiO}_{2}$ 的降解率明显低于其它 样品, $\mathrm{N}^{-\mathrm{TiO}_{2}}$ 光催化剂紫外光降解 $\mathrm{RhB}$ 的能力顺序 为 $2 \mathrm{~N}-\mathrm{TiO}_{2}>\mathrm{TiO}_{2}>1 \mathrm{~N}-\mathrm{TiO}_{2}>3 \mathrm{~N}-\mathrm{TiO}_{2}$, 当 $\mathrm{N}$ 的掺杂量 较少时, $2 \mathrm{~N}-\mathrm{TiO}_{2}$ 的紫外光催化活性略高于纯 $\mathrm{TiO}_{2}$, 当 $\mathrm{N}$ 含量为 $1.10 \%(x)$ 时, $3 \mathrm{~N}-\mathrm{TiO}_{2}$ 的紫外光催化活 性显著下降. 图 $4 \mathrm{~b}$ 为 $\mathrm{N}-\mathrm{TiO}_{2}$ 的可见光催化活性测 试结果, 从图中可以看出, $\mathrm{MO}$ 溶液经过 $4 \mathrm{~h}$ 可见光 催化降解后, $2 \mathrm{~N}-\mathrm{TiO}_{2}$ 的降解率最高, 样品的可见光 催化降解 $\mathrm{MO}$ 的能力顺序为 $2 \mathrm{~N}-\mathrm{TiO}_{2}>1 \mathrm{~N}-\mathrm{TiO}_{2}>3 \mathrm{~N}-$ $\mathrm{TiO}_{2}>\mathrm{TiO}_{2}$. 在可见光的照射下, $\mathrm{TiO}_{2}$ 几乎没有光催 化活性, 随着 $\mathrm{N}$ 掺杂量的增多, $\mathrm{N}-\mathrm{TiO}_{2}$ 的可见光降 解效率先增大后减小, $3 \mathrm{~N}-\mathrm{TiO}_{2}$ 的可见光降解活性 较弱. 以上结果说明 $\mathrm{TiO}_{2}$ 中掺人适量的 $\mathrm{N}$ 可以增 强其光催化活性, 而过量的 $\mathrm{N}$ 掺杂反而会降低其可 见光催化活性.

\section{3 催化剂的表面光电性质}

为了更深人地研究 $\mathrm{N}$ 的掺杂对 $\mathrm{TiO}_{2}$ 的光催化 性能的影响, 我们进行了表面光伏和瞬态光伏的测

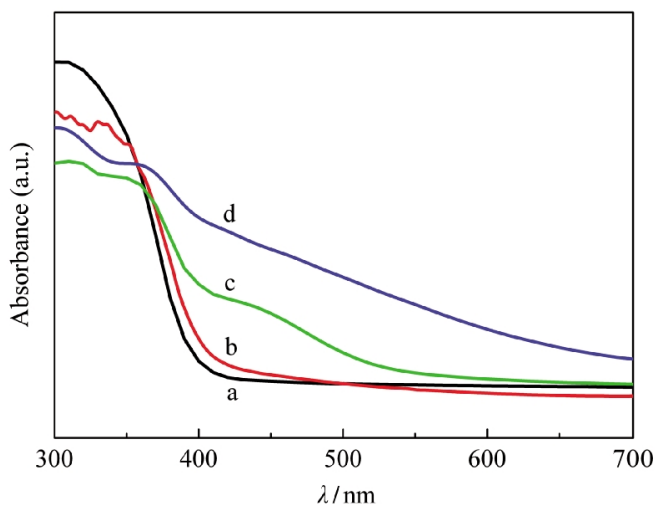

图 3 不同 $\mathbf{N}$ 掺杂量的 $\mathbf{N}-\mathbf{T i O}_{2}$ 纳米粒子的紫外-可见 漫反射谱图

Fig.3 UV-Vis diffuse reflectance spectra of $\mathbf{N}-\mathrm{TiO}_{2}$ nanoparticles with different $\mathbf{N}$-doping contents (a) $\mathrm{TiO}_{2}$, (b) $1 \mathrm{~N}-\mathrm{TiO}_{2}$, (c) $2 \mathrm{~N}-\mathrm{TiO}_{2}$, (d) $3 \mathrm{~N}-\mathrm{TiO}_{2}$
试. 图 5 为样品的表面光电压谱(SPS). 从 SPS 图中 可以看出, 与纯 $\mathrm{TiO}_{2}$ 不同, $\mathrm{N}-\mathrm{TiO}_{2}$ 紫外-可见光范围 内出现了两个明显的光伏响应带, 一个是与带带跃 迁相关的 $P_{1}$ 带 $(300-380 \mathrm{~nm})$, 一个是与亚带隙相关 的 $P_{2}$ 带 $(380-500 \mathrm{~nm})$. 在 $P_{1}$ 带, $2 \mathrm{~N}-\mathrm{TiO}_{2}$ 的响应强 度最大, 意味着光生电子-空穴对在表面空间电荷区 形成的自建场的作用下分离效率较高 ${ }^{[23]}$, 当 $\mathrm{N}$ 含量 增加到 $1.10 \%(x)$ 时, $3 \mathrm{~N}-\mathrm{TiO}_{2}$ 的光伏响应强度反而 降低, 这可能是因为过量的 $\mathrm{N}$ 以及氧空位都会成为 光生电子-空穴对的复合中心, 致使载流子复合几率 变大. 从图 6 样品的苂光光谱也可以看出, 在 $\mathrm{N}$ 含量 较低时,样品的苂光发射带强度随着 $\mathrm{N}$ 的掺杂而减 弱, 当 $\mathrm{N}$ 含量增大到 $1.10 \%(x)$ 时, $\mathrm{N}^{-\mathrm{TiO}_{2}}$ 的苂光强 度反而大于 $\mathrm{TiO}_{2}$, 说明适量的 $\mathrm{N}$ 掺杂有助于光生电 荷的分离, 过量的 $\mathrm{N}$ 掺杂可能使光生电荷在传输过 程中迅速复合. 结合图 4a 的紫外光催化结果, 样品 $2 \mathrm{~N}-\mathrm{TiO}_{2}$ 的紫外光催化活性比 $\mathrm{TiO}_{2}$ 的稍高, $3 \mathrm{~N}-\mathrm{TiO}_{2}$ 的催化活性最差, 这一结果与表面光伏测试基本一 致, 表明 $\mathrm{N}$ 掺杂过量时复合可能占主要作用. 而对
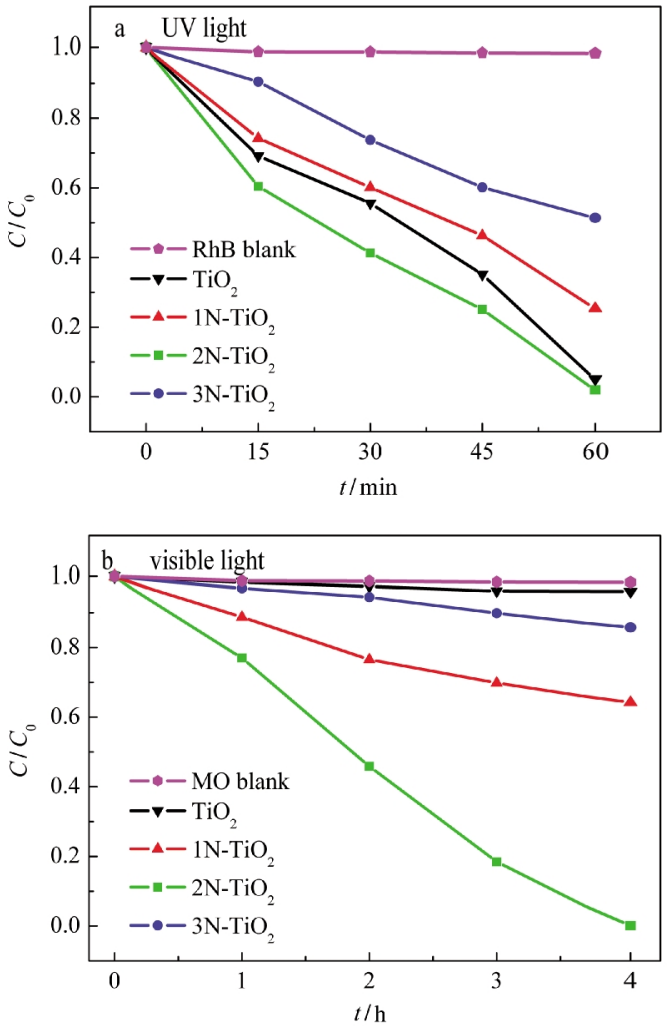

图 $4 \mathrm{~N}^{-\mathrm{TiO}_{2}}$ 光催化剂紫外光照射下降解罗丹明 $\mathrm{B}(\mathrm{a})$ 及可 见光 $(\lambda>420 \mathrm{~nm})$ 照射下降解甲基橙(b)的光催化降解曲线

Fig.4 Photodegradation curves of $\mathrm{RhB}$ under UV light (a) and MO under visible light ( $\lambda>420 \mathrm{~nm})(\mathrm{b})$ using $\mathrm{N}-\mathrm{TiO}_{2}$ as photocatalysts 


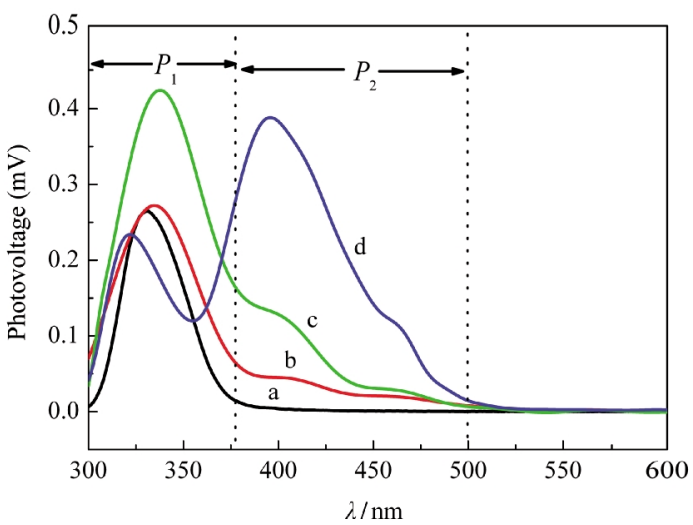

图 $5 \mathrm{~N}^{-\mathrm{TiO}_{2}}$ 纳米粒子的表面光电压谱图

Fig.5 Surface photovoltage spectra (SPS) of $\mathrm{N}_{-1} \mathrm{TiO}_{2}$ nanoparticles

(a) $\mathrm{TiO}_{2}$, (b) $1 \mathrm{~N}-\mathrm{TiO}_{2}$, (c) $2 \mathrm{~N}-\mathrm{TiO}_{2}$, (d) $3 \mathrm{~N}-\mathrm{TiO}_{2}$

$P_{2}$ 带, 随着 $\mathrm{N}$ 掺人量的增加, 其强度呈逐渐增大的 趋势, 其中, $3 \mathrm{~N}-\mathrm{TiO}_{2}$ 的表面光伏的强度最大, 而根 据图 $4 \mathrm{~b}$ 的结果, 其可见光催化活性反而最低. 我们 推测这是由于随着 $\mathrm{N}$ 掺人量的增多, 虽然提高了其 在可见光区光生电子-空穴对的分离效率, 但是当 $\mathrm{N}$ 掺杂过量时, 在 $\mathrm{TiO}_{2}$ 价带上方形成的 $\mathrm{N}$ 掺杂能级 向高能级处扩展 ${ }^{[24]}$, 使得在可见光的照射下, 从这些 掺杂能级产生的空穴的氧化能力会变弱, 从而降低 了可见光催化活性. 由于样品的光催化活性不仅与 载流子的分离有关, 还与载流子的传输以及复合过 程相联系. 为此, 我们进行了瞬态光伏的测试, 研究 其光生载流子的动力学信息.

图 7 为样品的瞬态光伏(TPV)谱图. 从图中可以 进一步得到纳米粒子中过剩的光生载流子的动力学 信息. 图中样品的激发波长为 $355 \mathrm{~nm}$, 激光脉冲的 强度为 $50 \mu \mathrm{J}$. 从 TPV 结果可以得出样品的光伏信

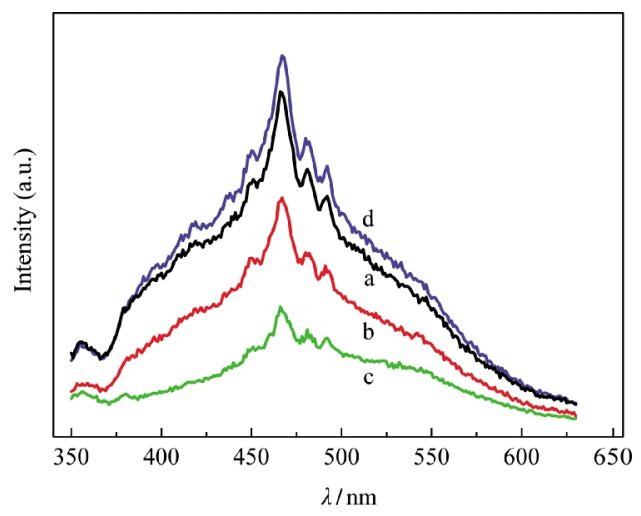

图 $6 \mathrm{~N}-\mathrm{TiO}_{2}$ 纳米粒子的荧光光谱图

Fig.6 Photoluminescence (PL) spectra for $\mathrm{N}_{-} \mathrm{TiO}_{2}$ nanoparticles

(a) $\mathrm{TiO}_{2}$, (b) $1 \mathrm{~N}-\mathrm{TiO}_{2}$, (c) $2 \mathrm{~N}-\mathrm{TiO}_{2}$, (d) $3 \mathrm{~N}-\mathrm{TiO}_{2} ; \lambda_{\text {ex }}=325 \mathrm{~nm}$

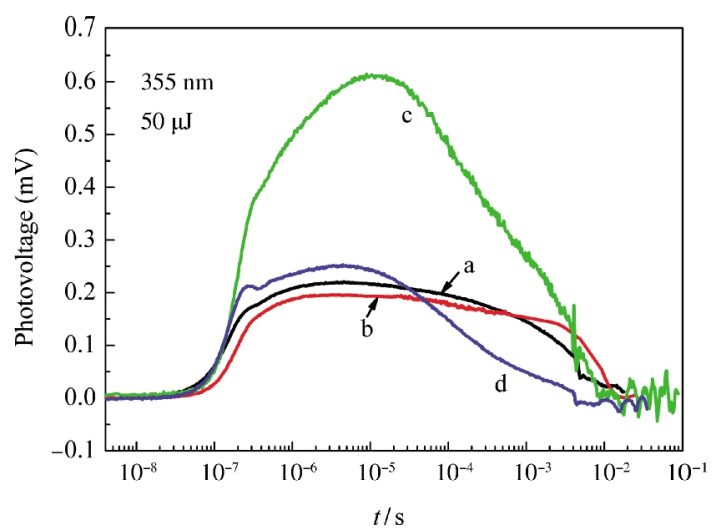

图 $7 \mathrm{~N}^{-\mathrm{TiO}_{2}}$ 纳米粒子的瞬态光伏谱图

Fig.7 Transient photovoltage (TPV) responses of $\mathrm{N}-\mathrm{TiO}_{2}$ nanoparticles

(a) $\mathrm{TiO}_{2}$, (b) $1 \mathrm{~N}-\mathrm{TiO}_{2}$, (c) $2 \mathrm{~N}-\mathrm{TiO}_{2}$, (d) $3 \mathrm{~N}-\mathrm{TiO}_{2}$

号均为正, 意味着光生空穴富集在照光电极一侧 ${ }^{[2]}$. 由于电荷的扩散速度远小于电荷在自建场下的漂 移, 所以可以认为, 在 $3 \times 10^{-7} \mathrm{~s}$ 内, 光生电荷在 $\mathrm{N}-$ $\mathrm{TiO}_{2}$ 颗粒内主要以漂移的方式进行分离和传输, 在 $3 \times 10^{-7} \mathrm{~s}$ 之后, 光生电荷的分离和传输主要在纳米 $\mathrm{N}-\mathrm{TiO}_{2}$ 颗粒间以扩散进行, 扩散的驱动力是光在样 品中的不均匀吸收而引起的光生电荷的浓度梯度. 从图中可以看出, 样品的光伏信号存在着显著的差 异. 对于样品 $2 \mathrm{~N}-\mathrm{TiO}_{2}$, 光伏信号在 $1 \times 10^{-5} \mathrm{~s}$ 时达到 最大值 $0.61 \mathrm{mV}$, 直到 $1 \times 10^{-2} \mathrm{~s}$ 复合完全; 而 $3 \mathrm{~N}-$ $\mathrm{TiO}_{2}$ 的样品, 光生电子-空穴对在 $5 \times 10^{-6} \mathrm{~s}$ 之后就开 始以复合为主, 到 $4 \times 10^{-3} \mathrm{~s}$ 复合完全; 样品 $1 \mathrm{~N}-\mathrm{TiO}_{2}$ 和 $\mathrm{TiO}_{2}$, 光生电荷在自建场作用下发生漂移后, 光 伏信号开始缓慢降低, 到 $1 \times 10^{-2} \mathrm{~s}$ 复合完全. 结果表 明 $2 \mathrm{~N}-\mathrm{TiO}_{2}(\mathrm{~N}$ 含量为 $0.71 \%(x)$ )样品的光伏响应达 到最大值的时间 $\left(t_{\text {max }}\right)$ 最长, 而且光伏响应最强, 意味 着与其它样品相比, $2 \mathrm{~N}-\mathrm{TiO}_{2}$ 的光生载流子的寿命 较长, 复合速率较慢, 表明光生电荷的分离效率较 高.

以上结果说明, 虽然不同 $\mathrm{N}$ 掺杂量的 $\mathrm{TiO}_{2}$ 样 品光生电荷的分离机制是类似的, 但是 $\mathrm{N}$ 的含量对 于 $\mathrm{TiO}_{2}$ 光生电荷的分离效率和复合速率有显著的 影响. 适量的 $\mathrm{N}$ 掺杂有助于光生电荷的分离, 过量 的 $\mathrm{N}$ 可能使光生电荷在传输过程中迅速复合, 降低 了光生载流子的寿命, 所以导致了样品 $3 \mathrm{~N}-\mathrm{TiO}_{2}$ 光 催化活性的下降.

\section{3 结 论}

通过对不同 $\mathrm{N}$ 掺杂 $\mathrm{TiO}_{2}$ 样品的光电性质和光 
催化活性的研究, 表明 $\mathrm{N}$ 的适量掺杂可以提高催化 剂中光生载流子的分离效率, 抑制其复合, 延长光生 载流子的寿命, 从而提高样品的紫外光及可见光催 化活性; 而过多的 $\mathrm{N}$ 掺杂以及氧空位将成为光生载 流子的复合中心, 增加了光生电子-空穴的复合几 率, 从而抑制其光催化活性.

\section{References}

1 Mor, G. K.; Shankar, K.; Paulose, M.; Varghese, O. K.; Grimes, C. A. Nano Lett., 2006, 6: 215

2 Konstantinau, I. K.; Albanis, T. A. Appl. Catal. B, 2003, 42: 319

3 Sakthivel, S.; Kisch, H. Angew. Chem. Int. Edit., 2003, 42: 4908

4 Hua, N. P.; Wu, Z. Y.; Du, Y. K.; Zou, Z. G.; Yang, P. Acta Phys. Chim. Sin., 2005, 21: 1081 [华南平, 吴尊义, 杜玉扣, 邹志刚, 杨 平. 物理化学学报, 2005, 21: 1081]

5 Asahi, R.; Morikawa, T.; Ohwaki, T.; Aoki, K.; Taga, Y. Science, 2001, 293: 269

6 Lin, Z. S.; Orlov, A.; Lambert, R. M.; Payne, M. J. Phys. Chem. B, 2005, 109: 20948

7 Kuroda, Y.; Mori, T.; Yagi, K.; Makihata, N.; Kawahara, Y.; Nagao, M.; Kittaka, S. Langmuir, 2005, 21: 8026

8 Kisch, H.; Sakthivel, S.; Janczarek, M.; Mitoraj, D. J. Phys. Chem. $C, 2007,111: 11445$

9 Yang, K. S.; Dai, Y.; Huang, B. B. J. Phys. Chem. C, 2007, 111: 12086

10 Tian, F. H.; Liu, C. B. J. Phys. Chem. B, 2006, 110: 17866

11 Zhou, Y. K.; Holme, T.; Berry, J.; Ohno, T. R.; Ginley, D.; Hayre,
R. O. J. Phys. Chem. C, 2010, 114: 506

12 Li, J. Y.; Lu, N.; Quan, X.; Chen, S.; Zhao, H. M. Ind. Eng. Chem. Res., 2008, 47: 3804

13 Linsebigler, A. L.; Lu, G. Q.; Yates, J. T. Chem. Rev., 1995, 95 735

14 Monllor-Satoca, D.; Gómez, R. J. Phys. Chem. C, 2008, 112: 139

15 Gross, D.; Mora-Seró, I.; Dittrich, T.; Belaidi, A.; Mauser, C.; Houtepen, A. J.; Como, E. D.; Rogach, A. L.; Feldmann, J. J. Am. Chem. Soc., 2010, 132: 5981

16 Duzhko, V.; Timoshenko, V. Y.; Koch, F.; Dittrich, T. Phys. Rev. $B$, 2001, 64: 075204

17 Alexander, D. Q.; Li, L. S. J. Phys. Chem. B, 2004, 108: 12842

18 Huang, D. G.; Liao, S. J.; Zhou, W. B.; Quan, S. Q.; Liu, L.; He, Z. J.; Wan, J. B. J. Phys. Chem. Sol., 2009, 70: 853

19 Sathishi, M.; Viswanathan, B.; Viswanath, R. P.; Gopinath, C. S. Chem. Mater., 2005, 17: 6349

20 Li, H.; Li, J.; Huo, Y. J. Phys. Chem. B, 2006, 110: 1559

21 Burda, C.; Lou, Y.; Chen, X.; Samia, A. C. S.; Stout, J.; Gole, J. M. Nano Lett., 2003, 3: 1049

22 Valentin, C. D.; Pacchioni, G.; Selloni, A.; Livraghi, S.; Giamello, E. J. Phys. Chem. B, 2005, 109: 11414

23 Kronik, L.; Shapira, Y. Surf. Sci. Rep., 1999, 371: 206

24 Liu, E. K.; Zhu, B. S.; Luo, J. S. Semiconductor physics. Beijing: Electronics Industry Press, 2003: 98-100 [刘恩科, 朱秉升, 罗晋 生. 半导体物理学. 北京: 电子工业出版社, 2003: 98-100]

25 Wei, X.; Xie, T. F.; Xu, D.; Zhao, Q. D.; Pang, S.; Wang, D. J. Nanotechnology, 2008, 19: 275707 